\title{
Lexis
}

Journal in English Lexicology

1 | 2008

Polysemy

\section{Les noms du pétrole : une approche diachronique de la métonymie onomastique}

\section{Pascaline Dury}

\section{(2) OpenEdition}

\section{Journals}

Édition électronique

URL : http://journals.openedition.org/lexis/749

DOI : $10.4000 /$ lexis.749

ISSN : 1951-6215

Éditeur

Université Jean Moulin - Lyon 3

Référence électronique

Pascaline Dury, "Les noms du pétrole : une approche diachronique de la métonymie onomastique », Lexis [En ligne], 1 | 2008, mis en ligne le 04 juillet 2008, consulté le 03 mai 2019. URL : http:// journals.openedition.org/lexis/749; DOI : 10.4000/lexis.749

\section{(c) (i) (3)}

Lexis is licensed under a Creative Commons Attribution-NonCommercial-NoDerivatives 4.0 International License. 


\section{Les noms du pétrole : une approche diachronique de la métonymie onomastique}

Pascaline Dury ${ }^{1}$

Mots-clés : Diachronie - langue de spécialité - métonymie onomastique et toponymique unité lexicale transitoire - synonymie de circonstance - enrichissement du sens provisoire

${ }^{1}$ Université Lumière - Lyon 2, CRTT (Centre de Recherche en Terminologie et Traduction). 


\section{Introduction}

La métonymie est une figure de rhétorique classique, bien connue et bien illustrée pour la langue générale, et l'on connaît depuis longtemps le rôle qu'elle joue, dans ce cadre, en matière d'enrichissement des unités lexicales. Cependant, la métonymie joue-t-elle exactement le même rôle lorsqu'elle se produit dans une langue de spécialité où la polysémie est plus redoutée que recherchée ? Une approche diachronique permet-elle d'envisager la métonymie sous un angle différent, en montrant notamment son importance pour la constitution d'un lexique spécialisé ?

Nous nous proposons d'apporter quelques éléments de réponse à ces questions, en nous appuyant sur une étude des noms anglais du pétrole, menée sur un corpus diachronique contenant des articles et des ouvrages spécialisés du XIXe siècle à nos jours.

Nous verrons d'abord quels étaient les noms du pétrole au XIXe, et pourquoi la plupart d'entre eux peuvent être considérés comme des unités lexicales concurrentes et provisoires ; nous soulignerons ensuite l'importance de la métonymie onomastique et notamment toponymique dans les mots qui désignent le pétrole à cette époque, et dans une dernière partie, nous étudierons le rôle qu'elle a pu jouer dans la construction de ce lexique.

\section{Définition de la problématique et description du corpus utilisé}

\subsection{Définition de la problématique}

Des travaux antérieurs dans le domaine de la terminologie, menés sur la langue de l'écologie (Dury [1996, 2007]), nous avaient amenée à faire certaines observations sur l'adaptation d'une langue de spécialité à l'évolution du savoir scientifique et nous avaient permis d'observer, pour quelques termes bien précis, comment à un signifié nouveau venait s'attacher un signifiant nouveau. Le travail mené autour des mots du pétrole et présenté dans cet article contribue à notre réflexion sur la création des signes linguistiques et la constitution des lexiques spécialisés. La problématique de ce travail repose plus particulièrement sur l'observation, en diachronie, des termes formés sur le principe de la métonymie (notamment onomastique) qui apparaissent dans un lexique spécialisé comme celui du pétrole. Observée sous cet angle-là, la métonymie remplit-elle la même fonction que dans la langue générale et les nouveaux sens produits par métonymie ainsi que les unités lexicales qui les expriment s'implantent-ils durablement dans le lexique étudié ?

\subsection{Terminologie utilisée}

Au cours de cet article, nous utiliserons la terminologie proposée par Jean Tournier au sujet de la «métasémie », qu'il désigne comme «le véritable changement de sens [...], qui est indépendant d'un changement de fonction du mot» [2004: 127]. Le phénomène de changement de sens par métonymie fait partie des mécanismes de la métasémie et se fonde, non pas sur la perception d'une ressemblance, mais «sur une association d'idées 》 (Tournier [2004 : 145]). Nous avons donc étudié, dans une perspective diachronique, tous les termes produits par ce type de mécanismes et pour lesquels on pouvait observer un glissement de référence d'un objet à un autre. Nous avons tout particulièrement porté notre attention sur la 
métonymie onomastique (Tournier (2004) parle d'« antonomase »), qui s'appuie sur l'association d'idées avec un nom propre, et dans le cas du lexique du pétrole, plus particulièrement avec un nom de lieu (métonymie toponymique).

\subsection{Description du corpus et choix de la période étudiée}

Cet article se base sur l'exploitation d'un corpus construit à partir d'une recherche menée autour du terme petroleum, dans le domaine de la minéralogie et de la géologie. Il s'agit d'un corpus de langue anglaise, ouvert, diachronique, et spécialisé qui contient environ 150000 mots issus d'ouvrages et d'articles du domaine allant du xixe à nos jours.

Cette délimitation chronologique repose sur des motifs d'ordre historique et d'ordre linguistique. Sur le plan du mouvement de la société, la période choisie offre un contexte historique, social et économique caractérisé, avant tout, par un puissant essor des techniques et par leur application à la grande industrie, alors en plein épanouissement. La géologie du pétrole représente pourtant, à l'époque qui nous intéresse, un véritable paradoxe : le produit existe bien entendu à l'état naturel depuis quelque 5000 ans déjà et est utilisé, exploité localement, à petite échelle, et même vendu dans quelques pays et régions d'Europe (Roumanie, Pologne, Ecosse, Italie, Allemagne, France), au Moyen-Orient et aux Etats-Unis, mais il ne connaît pourtant pas encore l'essor industriel qui se produit à l'époque dans d'autres domaines. Selon Owen [1975: 1] :

One of the world's most essential industries was left to grow up in the backwoods of Pennsylvania. The fact that the cumulative experience became productive at this location- and that the ancient tradition did not mature until this time (1859), constitutes one of the great paradoxes of economic history.

Il faut en fait attendre la deuxième moitié du XIXe pour que l'industrie du pétrole connaisse un développement radical, notamment grâce à la découverte et à l'exploitation d'un gisement de grande taille, le 28 août 1859, à Oil Creek, près de Titusville, petite ville de Pennsylvanie. Il semblerait que le lieu (et le nom qu'il porte en est une bonne indication) était déjà connu pour le pétrole que l'on pouvait y trouver, mais personne n'en avait encore exploité les ressources à grande échelle.

Les premiers missionnaires jésuites qui explorent la région au XVIIe mentionnent la présence de pétrole à Oil Creek, pétrole alors utilisé par les Indiens Seneca pour la préparation de médecines traditionnelles ${ }^{2}$. Peter Kalm, étudiant de Linné, visite l'endroit en 1749 et décrit le pétrole qu'on y trouve dans le récit de son voyage. Les gisements de pétrole d'Oil Creek sont même indiqués sur les premières cartes géographiques de l'Amérique coloniale que Lewis Evans réalise en 1755. En 1854, George H. Bissel et Jonathan G. Eveleth de New York achètent les parcelles de terrain contenant le pétrole et fondent la Rock Oil Company, espérant tirer profit de l'exploitation et de la vente d'un produit dont une publication de Benjamin Silliman (1833), très remarquée au moment de sa parution et dans les années suivantes, avait vanté les multiples qualités. L'affaire ne s'avère cependant pas rentable et la Rock Oil Company devient alors la Seneca Oil Company, dont le « Colonel » Drake 3 prend la direction. C'est alors que le 28 août 1859, un puits de pétrole, qui vient juste d'être creusé à même la

\footnotetext{
${ }^{2}$ Les informations qui suivent sont tirées de Forbes (1959).

${ }^{3}$ La légende née autour du « Colonel » Drake a quelque peu embelli la vérité historique et veut que ce dernier, en lançant son chapeau dans les airs, trouva l'emplacement de ce fameux premier puits d'où jaillit le pétrole, là où le chapeau était retombé.
} 
roche, révèle un gisement d'une grande importance, situé à environ 23 mètres de profondeur : c'est le début de la ruée vers l'or noir".

Une situation privilégiée sur le plan linguistique nous est ainsi offerte : il s'agit d'observer comment, à l'aube de l'essor de l'industrie moderne du pétrole, le lexique de ce domaine rend compte des difficultés à exprimer des connaissances scientifiques encore floues et désigne un produit malgré tout encore mal connu, dont l'exploitation à grande échelle n'a pas encore tout à fait débuté.

\section{Les mots du pétrole au XIXe : une concurrence entre unités lexicales provisoires}

\subsection{Foisonnement néologique transitoire et synonymie de circonstance}

Nous l'avons vu, le pétrole est un produit connu depuis près de 5000 ans, qui a été utilisé par de nombreuses civilisations au cours des siècles, à des fins multiples : il était notamment employé comme élément de construction et pour protéger le bois contre les insectes, pour s'éclairer $^{5}$, mais aussi pour se soigner, puisqu'on lui attribuait de nombreuses vertus médicales. D'ailleurs, Farey [1811: 468] note : "The miners of Derbyshire apply liquid Bitumen or Rock-Oil to fresh wounds, with great success, towards their cure ».

On constate cependant que même si le produit est connu depuis un certain temps déjà, les avis scientifiques, au moins pendant quelque temps et en particulier au XIxe, puisque c'est la période qui nous intéresse ici, divergent sur l'essentiel : son origine, de quoi il est composé, quels produits dérivés on peut en tirer, et aussi ce à quoi il peut servir exactement. Malgré les difficultés bien connues de faire un rétrodiagnostic, et les risques qu'il y a toujours à enjamber la chronologie, on peut supposer que c'est justement cette relative imprécision des connaissances au sujet du pétrole qui s'est traduite, au niveau du lexique, par la coexistence et la mise en concurrence d'unités lexicales provisoires, décrivant, dans la plupart des cas, le même produit, mais avec des noms différents. Guilbert dans son observation des vocabulaires en gestation évoquait déjà l'existence de ce phénomène, auquel il donna le nom de foisonnement néologique transitoire [1965: 331] :

Dans la période de création d'une réalité nouvelle et de formation d'un vocabulaire adéquat, c'est une caractéristique de la situation linguistique qu'un certain foisonnement néologique transitoire se produise pour désigner un même concept.

Ce foisonnement néologique transitoire, caractéristique des lexiques en formation et décrit par Guilbert (1965) pour le domaine de l'aviation, se manifeste par une production accrue de néologismes concurrents, coexistant pour quelque temps dans le lexique en question, puis disparaissant de ce lexique ou se spécialisant d'un point de vue sémantique pour continuer d'exister.

\footnotetext{
${ }^{4}$ On peut remarquer au passage que plus de la moitié des ouvrages publiés au XIXe sur le pétrole et utilisés pour la constitution de notre corpus sont parus après 1859 (17 ouvrages sur 25), ce qui confirme l'importance de la date en question, qui correspond indubitablement à l'essor industriel du pétrole.

${ }^{5}$ Selon Owen [1975: 2] : In Burma [petroleum] was used in house construction and boat building, principally as wood preservative, insect repellent, and waterproofing. All families within reach of water transportation used the oil as an illuminant.
} 
Dans le cas du lexique étudié ici, il est intéressant d'observer que chez la plupart des auteurs, ce foisonnement se manifeste par la mise en concurrence provisoire de néologismes tout autant que de termes existant déjà dans le domaine, mais aux contours sémantiques assez flous, qui deviennent alors concurrents, de façon transitoire, pour désigner le pétrole. Nous les appellerons, comme nous le verrons plus loin, des « synonymes de circonstance ».

\title{
2.2. Qu'est-ce que le pétrole, d'où vient-il et à quoi sert-il ?
}

Il faut souligner à quel point l'utilisation des termes désignant le pétrole à cette époque peut sembler anarchique et aléatoire, puisque chez certains auteurs petroleum est bien synonyme de naphtha, bitumen, oil, mineral oil et tar, alors que chez d'autres, il s'agit de produits différents, d'ailleurs considérés parfois comme étant issus du pétrole lui-même. C'est le cas notamment pour le terme naphtha:

\footnotetext{
Near London, the naphtha or petroleum is found floating on the surface of the etangs, or stagnant waters of the Thames, and is frequently collected by means of a piece of cloth. Taylor [1848: 185]
}

Petroleum, or rock-oil, is found in various parts of the world [...]. It consists of a combination of carbon and hydrogen, and from it naphtha and paraffin are sometimes derived. Transactions of the Manchester Geological Society [1862 : vol.3, 14]

Il arrive parfois que l'ambiguïté d'emploi qui caractérise ces termes se produise chez un même auteur, et que deux termes qui entrent en concurrence synonymique dans un paragraphe soient définis comme désignant des produits différents quelques pages plus loin. On trouve ainsi dans l'ouvrage de Wells les descriptions suivantes du pétrole, qui montrent à quel point le sens des termes qui nous intéressent semble pour le moins « élastique » :

\footnotetext{
Notes on the history of petroleum or rock oil : the following comprehensive resumé gives the most important facts thus far made known respecting the geological history of the various substances designated respectively as « petroleum », " naphtha », « asphalt» and « mineral pitch ». David Wells [1862: 283]
}

\begin{abstract}
The form in which it now occurs depends in great measure upon the presence or absence of atmospheric oxygen, since by oxidation and volatilization what is called naphtha or petroleum, as we have already explained, become slowly changed into asphalt or mineral pitch, which is solid at ordinary temperature. David Wells [1862: 285]
\end{abstract}

On remarque au passage que les termes naphtha et oil (mais c'est le cas pour d'autres termes qui apparaissent dans le corpus, comme bitumen, tar et pitch, par exemple) existent déjà dans le vocabulaire du pétrole, avec des sens proches, et qu'un besoin ponctuel de dénomination provoque un glissement sémantique, les transformant alors, provisoirement, chez certains auteurs, en synonymes de circonstance. Il s'agit donc ici d'un enrichissement du lexique qui n'a qu'un caractère ponctuel et provisoire.

Cette «élasticité » du sens et cette synonymie de circonstance peuvent s'expliquer parce que même si les connaissances chimiques et minéralogiques au XIXe sont déjà importantes, elles ne sont cependant pas encore suffisamment avancées, semble-t-il, pour déterminer avec 
précision la composition exacte du pétrole, son origine et ses propriétés ${ }^{6}$. Dans son ouvrage sur l'histoire de l'exploration du pétrole, Owen [1975: 17-18] note :

\begin{abstract}
Many, perhaps most, geologists, mineralogists, and chemists during the first half of the 19th century took at least casual notice of occurrences of oil and gas and some of the implied problems. They knew already that the substances had some economic value, that their qualities were varied although the principal ingredients were basically carbon and hydrogen, and that they reached the surface of the earth at many places in circumstances which were in some ways peculiar. Something was known of the physics of fluids and heat and other fundamental matters. Most of the gross geographic and geologic features had been explored superficially. But the scientist's comprehension was circumscribed by limited knowledge of stratigraphy, structure, and precise geologic and chemical processes.
\end{abstract}

Ainsi, chez les deux auteurs suivants, le pétrole est dans le premier cas présent dans la roche et issu de la décomposition d'organismes marins (animaux et végétaux), alors que dans le deuxième cas, le pétrole se trouve dans la tourbe et est décrit comme étant le produit d'une lente fermentation végétale :

The substance, which is doubtless of organic origin, occurs in rocks of all ages [...] and its presence in the lower palæozoic rocks, which contain not traces of land plants, shows that it has not in all cases been derived from terrestrial vegetation, but may have been formed from marine plants and animals; the latter is not surprising when we consider that a considerable portion of the tissues of the lower marine animals is destitute of nitrogen, and very similar in chemical composition to the woody fibre of plant. David Wells [1862: 286]

These circumstances, added to the fact of the petroleum being found most plentifully at the edge of the sand, led to the conclusion that it is produced by the decomposition of the upper bed of peat, where it is overlaid by sand: all that need to be said is that petroleum is probably the result of a slow fermentation or combustion of the vegetable matter composing the peat, and has been produced in a manner somewhat analogous to the hydro-carbons obtained by the destructive distillation of wood in close vessels. E.B. Andrews [1861: 92]

\title{
3. Importance de la métonymie toponymique
}

\subsection{Un grand nombre d'unités lexicales formées sur le principe de la métonymie}

Outre un foisonnement néologique caractéristique et une certaine élasticité d'emploi des termes ayant des sens proches, il nous est apparu que l'imprécision conceptuelle qui vient d'être évoquée se traduisait aussi par l'utilisation d'un grand nombre d'unités lexicales formées sur le principe de la métonymie utilisant les noms des régions ou des pays où l'on trouve du pétrole. La plupart des termes extraits du corpus sont particulièrement transparents et décrivent clairement ce qui est le plus directement observable dans le produit : notamment son origine, autrement dit où on le trouve dans la nature (rock oil, mineral pitch, earth oil), ce qu'il sent (empyreumatic oil), quel aspect il a (liquid bitumen, dark pitch, tar, carbon oil) et aussi dans quel pays ou quelle région on le trouve. Sur ce dernier point, on dénombre ainsi, pour désigner le pétrole, pas moins de 14 termes, construits sur le principe de la métonymie

\footnotetext{
${ }^{6}$ En guise d'illustration, une description chimique et minéralogique du pétrole, tirée de l'ouvrage de Robert Jameson (1820) est donnée dans l'annexe 2 de cet article.
} 
toponymique : Sicilian oil, Barbadoes tar, Barbadoes petroleum, Persian rock oil, Gabian oil, Genesee oil, Trinidad pitch, Trinidad asphalt, Trinidad bitumen, Seneca oil, Seneca rock oil, American earth oil, Rangoon petroleum, Rangoon earth oil.

Dans la totalité de notre corpus de 150000 mots, nous avons repéré 36 termes pour désigner le pétrole, dont plus d'un tiers peuvent être considérés comme étant formés sur le principe de la métonymie toponymique ${ }^{7}$ :

Petroleum, known as Barbadoes petroleum, or Barbadoes tar, or sometimes Barbadoes, the springs of which were visited by the author in 1818, still send forth their oily products, and the springs of other West India Islands and South America have not failed. Gesner [1861: 74]

Petroleum is a bituminous substance known under the name of Seneca or Genesee oil or Genesee and $[\ldots]$ is much used by farmers, and has a ready sale. Richard C. Taylor [1848: 137]

\subsection{Le cas de Seneca oil}

On notera cependant que pour l'un de ces composés métonymiques, Seneca oil, les auteurs divergent sur l'origine du terme Seneca. Pour certains il s'agit du nom que portait la tribu d'Indiens qui récoltait le pétrole à des fins médicales et qui vivait sur les bords d'un lac situé dans l'Etat de New York; pour d'autres en revanche, il s'agit du nom du lac en question.

[...] and even on the banks of the Mackenzie river, springs of naphtha have been found. It was long collected for sale from the Seneca lake, in New York, by the Indians, and sold under the name of Genesee or Seneca oil. The Technologist [1861: 244]

Considerable quantities of petroleum are also obtained in the States of Ohio, Kentucky, and New York, and is known under the name of Genesee or Seneca oil, from its having been formerly collected by the Seneca Indians. J. Sproule [1854: 89]

Il est un peu difficile dans ce cas de faire la part entre métonymie toponymique et métonymie anthroponymique car pour certains le nom Seneca Indians vient du lac autour duquel ils vivaient, alors que pour d'autres, c'est cette tribu précisément qui a donné son nom au $\operatorname{lac}^{8}$.

\subsection{Enrichissement provisoire du lexique}

La plupart des termes extraits de notre corpus semblent n'avoir appartenu au lexique du pétrole que de façon ponctuelle. En effet, la plus grande partie des termes décrivant l'origine minéralogique du produit (earth oil, rock oil, mineral pitch, mineral fish oil, etc.) ainsi que la totalité des termes construits sur le principe de la métonymie toponymique ont aujourd'hui

\footnotetext{
${ }^{7}$ Voir annexe 1.

${ }^{8}$ On retrouve la même ambiguïté dans la version en ligne de l'Oxford English Dictionary qui, pour l'entrée Seneca, donne les citations suivantes :

[Seneca oil, a name for crude petroleum: now only Hist.

1833 in Amer. Jrnl. Sci. XXIV. 64. This oil acquired its name of Seneca oil, from having been first found in the vicinity of Seneca Lake, N. York.

a1864 GESNER Coal, Petrol., etc. (1865) 16 Under the name of 'Seneca Oil' which it derived from an Indian tribe, petroleum was formerly collected in Chatauque County, N.Y., and in Crawford County, Pennsylvania, and sold for medicinal purposes].
} 
disparu de la terminologie du domaine. Peu de termes sont restés, comme naphtha, asphalt, bitumen, maltha, tar et pitch, mais tous ont vu leur sens se spécialiser pour continuer d'exister. Ainsi le terme asphalt décrit aujourd'hui un composé naturel ou artificiel d'hydrocarbures issu de la distillation du pétrole, qui sert à divers usages industriels et techniques, notamment pour le revêtement des routes. L'Oxford English Dictionary en donne la définition suivante :

1. A bituminous substance, found in many parts of the world, a smooth, hard, brittle, black or brownish-black resinous mineral, consisting of a mixture of different hydrocarbons.

2. A composition made by mixing bitumen, pitch and sand, or manufactured from natural bituminous limestones, used to pave streets and walks, to line cisterns, etc.

De même, on emploie désormais le terme maltha pour décrire un bitume glutineux, moins liquide que le pétrole ${ }^{9}$. D'un point de vue diachronique, il ne s'agit donc bien que d'une néologie sémantique provisoire ou de circonstance, car des termes considérés ponctuellement comme synonymes au XIXe siècle ont aujourd'hui des sens bien distincts.

\section{Synthèse et conclusion}

Nous constatons donc que l'imprécision des connaissances caractéristique du domaine du pétrole au XIXe, période à laquelle il ne connaît pas encore une industrialisation à grande échelle et ne suscite donc pas encore l'intérêt massif des scientifiques et des professionnels, s'exprime au niveau lexical de deux façons :

1) Par un foisonnement néologique et en particulier une élasticité sémantique marquée pour des termes existants déjà dans le lexique en question, et se transformant ponctuellement en synonymes de circonstance, participant ainsi à un enrichissement provisoire du lexique,

2) Par une utilisation accrue de métonymes toponymiques, participant à la construction du lexique du pétrole, mais disparaissant de ce lexique lorsque les concepts se fixent et se précisent. Nous posons donc l'hypothèse suivante : les termes construits sur le principe de la métonymie toponymique abondent dans le vocabulaire du pétrole de cette époque, car ils répondent aux besoins qu'a toute communauté scientifique de décrire, de transmettre et de communiquer un savoir.

Or la métonymie toponymique donne une information sur le produit - dans quel(s) pays ou région(s) on le trouve - qui, même si elle est secondaire ou accidentelle, représente néanmoins un élément de connaissance stable et indiscutable, à une période où, comme nous l'avons souligné, il y a une relative imprécision scientifique de ce qu'est exactement le

\footnotetext{
${ }^{9}$ Pour les termes naphtha et maltha, l'Oxford English Dictionary note :

Naphtha: A volatile, flammable liquid obtained by the fractional distillation of petroleum or the destructive distillation of coal tar, shale, lignite, etc., and composed of a mixture of hydrocarbons; spec. such a distillate as used for making petrol, kerosene, and solvents, with a boiling point below about $200^{\circ} \mathrm{C}$. Also: a solvent, etc., made from this.

Rq: L'Oxford English Dictionary note que naphtha comme synonyme de liquid petroleum est aujourd'hui seulement d'un emploi littéraire ou poétique.

Maltha: A kind of cement made by mixing pitch or lime with wax, fat, or sand, and other ingredients.
} 
pétrole. On peut ensuite supposer que les termes construits sur le principe de la métonymie toponymique disparaissent du lexique, parce que la connaissance qu'ont les scientifiques du produit s'améliore, se précise, et que l'expression de ce caractère accidentel que représente l'origine géographique du produit devient alors superflue, voire obsolète.

D'un point de vue diachronique on peut donc considérer que la métonymie, notamment toponymique, a participé à la genèse du vocabulaire du pétrole au XIXe et peut être considérée, elle aussi, comme un instrument d'enrichissement - même provisoire - du lexique.

\section{Remerciements}

Merci à Henri Béjoint, Yannick Brolles et Claire Rougemont du CRTT pour leurs précieux conseils et leurs relectures attentives.

\section{Références}

Bachelard Gaston, La formation de l'esprit scientifique, Paris, Vrin, 1996.

Bonнomme Marc, Le Discours métonymique, Berne, Lang, 2006.

Dury Pascaline, Etude comparative et diachronique de l'évolution de dix dénominations fondamentales $d u$ domaine de l'écologie en anglais et en français, Presses Universitaires du Septentrion, 1997.

---, « La dimension diachronique en terminologie et en traduction spécialisée : le cas de l'écologie », Aspects diachroniques du vocabulaire, sous la direction de François Gaudin et Danielle Candel, Presses Universitaires de Rouen, 2006: 109-124.

EIA (Energy Information Administration), Petroleum, an Energy Profile 1999, Diane Publishing, 1999.

Forbes R.J., More Studies in Early Petroleum History 1860-1880, Leiden, E.J. Brill, 1959.

Gevaudan Paul, «Fondements sémiologiques du modèle de la filiation lexicale », Philologie im Netz, Volume 22, 2002: 1-26.

Guilbert Louis, La formation du vocabulaire de l'aviation, Paris, Librairie Larousse, 1965.

---, La créativité lexicale, Paris, Larousse Université, 1971.

Kalm Peter, The America of 1750: Peter Kalm's Travels in North America, New York, Adolph B. Benson, 2 volumes, 1966 [1933].

Owen Edgar Wesley, Trek of the Oil Finders: a History of Exploration for Petroleum, Tulsa, American Association for Petroleum Geologists, 1975.

PAILlARD Michel, Lexicologie contrastive anglais-français, formation des mots et construction de sens, Ophrys, 2000.

Toledano Violette et Danielle Candel, «Dérivation suffixale de toponymes : étude d'un terrain propice à la création lexicale », Méta, Volume 47, $\mathrm{n}^{\circ}$ 1, 2002: 105-124.

TOURNIER Jean, Introduction descriptive à la lexicogénétique de l'anglais contemporain, ParisGenève, Champion-Slatkine, 1985. 
---, Précis de lexicologie anglaise, Paris, Ellipses, 2004.

\section{Ouvrages issus du corpus et cités dans l'article}

Andrews E.B., "Rock Oil, its Geographical Relations and Distribution", The American Journal of Science and Arts, Volume 32, $\mathrm{n}^{\circ}$ 2, 1861: 85-93.

FAREY John, General View of the Agriculture and Minerals of Derbyshire, with Observations on the Means of their Improvement, London, MacMillan, 1811.

Gesner Abraham, A Practical Treatise on Coal, Petroleum and Other Distilled Oils, New York, Baillière Brothers, 1861.

Jameson Robert, A System of Mineralogy in which Minerals are Arranged according to the Natural History Method, Edinburgh, Archibald Constable \& Co., 1820.

Silliman Benjamin, "Notice of a Fountain of Petroleum, called the Oil Spring", American Journal of Science, Volume 23, 1833: 97-102.

Simmonds Peter Lund, éd., The Technologist, a Monthly Record of Science Applied to Art and Manufacture, London, Kent \& Co., Volume 1, 1861.

Sproule, John éd., The Irish Industrial Exhibition of 1853: a Detailed Catalogue of its Contents, Dublin, James McGlashan, London, William S. Orr and Co., 1854.

TAYlor Richard C., Statistics of Coal, Geographical and Geological Distribution of Mineral Combustables, Philadelphia, J.W. Moore, 1848.

Transactions of the Manchester Geological Society, Simpkin, Marshall and Co., Volume 3, 1862.

Wells David A., éd., Annual of Scientific Discovery or Year-Book of Facts in Science and Art for 1862, Boston, Gould and Lincoln, 1862. 
ANNEXE 1 : Liste des unités lexicales présentes dans le corpus et désignant le pétrole

\section{Petroleum or petrol}

Termes décrivant l'origine minéralogique du pétrole

Earth oil

Mineral naphtha

Mineral oil / Mineral fish oil

Mineral pitch

Rock oil / native rock oil

Rock pitch

Termes décrivant la " substance " ou «l'aspect " du pétrole

Asphalte/asphaltum

Bitumen

Carbon oil

Crude oil

Dark pitch

Liquid bitumen

Maltha

Naphtha

Oil

Tar

Termes décrivant l'odeur du pétrole

Empyreumatic oil

Termes décrivant " d'où vient " le pétrole

Native oil

Native naphta

Native paraffine oil

Métonymes toponymiques

Barbadoes tar/petroleum

Gabian oil

Genesee oil

Persian rock oil

Rangoon petroleum/earth oil

Seneca oil / Seneca rock oil

Sicilian oil

Trinidad pitch /asphalt/bitumen 
ANNEXE 2 : Extrait de l'ouvrage de Robert Jameson, A System of Mineralogy in which Minerals are Arranged according to the Natural History Method, 1820

and. 1. grsix.]

BY. 2. hLACK MTSERAL ResiN.

483

[Subop. 2. Mineral Oil, or Petroleum.

varnish; and formerly it was employed as a vermifuge medicine.

Second Subspecies.

Mineral Oil, or Petroleum.

Erdöl, Werner.

Bitumen liquidum, Plin. Hist. Nat. xxxv--Maltha tarde fluens, Wall. t. ï. p. 92.-Petrole, Rome de Lisle, t. ii. p. 591. Id. De Born, t. ii. p. 75.-Petrol, Kirv. vol. ii. p. 43. Id. Estner,

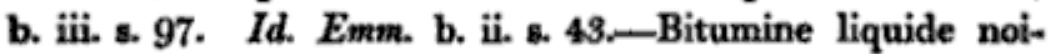
râtre, Haüy, t. iii. p. 812.-L'Huile minerale commune, ou le Petrol, Broch. t. ii. p. 59, 60-Gemeines Bergöl, Reuss, b. iii. s. 96,-101. Id. Mohs, b. ii. s. 302. Id. Leonhard, Tabel. s. 48-Bitumen Petrole, Brong. t. ii. p. 24-Verdicktes Bergö, Karsten, Tabel. s. 58.-Steinöl, Haus. s. 117 . -Petroleum, Kid, vol. ii. p. 62.-Bergöl, Lenz, b. ii. s. 1047. -Flussiges Bergtheer, Haus. Handb. b. i. s. 89.-Erdal, Hoff. b. iii. . 266.-Petroleum, Aikin, p. 59.

\section{External Characters.}

Its colour is dark blackish-brown, which sometimes inclines to green.

It is fluid, but approaches more or less to the viscid state.

It is shining and resinous.

It feels greasy.

It is semi-transparent, translucent, and opaque.

It exhales a strong bituminous odour.

It is so light as to swim on water.

Chemical Characters.

It inflames easily, emits a bluish flame, and yields a smoke 
GEN. Q. MTNERAL RESTX. [CL.4. TNYLAM: MUS.

smoke more or less opaque, according to the density of the oil, and sometimes leaves a very small earthy residue.

Constituent Parts.

It is composed of Carbon, Hydrogen, and a little Oxygen.

\section{Goognostic Situation.}

It generally flows from rocks of the coal formation, and usually from the immediate vicinity of beds of coal; also from limestone rocks. It occurs in marshes, on the surface of spring water; or it flows or trickles unmixed from its repository.

\section{Geographic Situation.}

Europe-Oozing from secondary rocks at St Cathe rine's Well, near Edinburgh, and in the island of Pomons, one of the Orkneys. Filling cavities and veins in limestone at Pitchford and Madeley in Shropshire. 'Several springs of this mineral occur in France, as at Gabian in Herault; in Auvergne; and at Pechelbrunn in Alsace, the sandstone is very highly impregnated ' with it. It is also found on the Lake Tegern, in Bavaria ; near Neufschintel in Switzerland; at Amiano, twelve leagues from Parmi; in Mount Zibio, near Modena; and a spring has been seen rising from the bottom of the sea in the Bay of Naples, which pours out much mineral oil. It is also met with in Sicily; in the salt-mines in Transylvania ; in Gallicia ; and in Moldavia, springs of petroleum flow from a track where there is an alternation of beds of sandstone, marl, gypsum, and rock-salt. 